\title{
Counting Method for Measuring and Linearity Checking Photometry Devices
}

\author{
E. Kuvaldin \\ Vavilov State Optical Institute the Russian Federation State Scientific and Manufacturing Corporation 12, \\ Birgevaya str., Saint Petersburg, 199034, RussiaＥ-mail: ekuvaldin@yandex.ru
}

\begin{abstract}
The possibility of calibration and linearity check of optical detectors in a wide dynamic range by method of counting separate parts of energy is investigated. Counting method permits nonselective energy change of measuring optical pulse more then $10^{5}$ times. A construction of working source of radiation with GaAs LED is described. Results of experimental investigation are presented.
\end{abstract}

Keywords: Optical measurement, check linearity, energy meter, calibration, pulse count

\section{INTRODUCTION}

$I^{T}$ $\mathrm{T}$ IS KNOWN that lasers and pulse lamps have very unstable power and energy from pulse to pulse. So it is very difficult to measure such sources. Method of averaging of pulse energy in series of $10,100,1000$ pulses permits us to decrease instability of measurements. For this purpose a device for measuring the energy of each pulse was designed. The readout of this device was made as a counter. Thus, one can easily know the average value from 10, 100, 1000 and so on pulses by moving the comma to the left by one digit for each tenfold increase of counting pulses. Such device can be calibrated by calorimetric standard device with a large time constant. For instance, if we have a series of 1000 pulses with common energy $10^{-3} \mathrm{~J}$ measured by a calorimeter, a single pulse will have energy $10^{-6} \mathrm{~J}$ and device readout will be $10^{-6} \mathrm{~J}$ as average from 1000 pulses. It will be a rather stable value. So we can precisely decrease energy of measuring pulse in $10^{3}$ times if we use only one pulse for measuring. High sensitivity pyroelectric energy meters were calibrated earlier in such manner.

\section{COUNTING METHOD}

The next modification of the calibration method uses pulse source with changing energy in each measuring pulse. Decreasing or increasing energy in each pulse and in series of pulses can be made in different ways. The most popular is changing of pulse duration when a period between pulses is constant. It can be made into an analog way by changing duration of each pulse with a constant period of time between pulses, but better if the length of each pulse in a measuring series of pulses is filled by a number of short counting pulses with a stable energy in each counting pulse. The waveform of the counting pulse does not matter. If you change a number of short counting pulses for the duration of pulses in the measuring series, you can change energy in each pulse and in the whole series. In a series of 100 measuring pulses, for example, with a repetition rate of $100 \mathrm{~Hz}$ and common length of 1 second, short counting pulses with duration less than 1 microsecond are situated within the duration of each measuring pulse. It is very useful to have an interval between pulses for illuminating source working in good temperature conditions. More power and energy can be received in each measuring pulse in this case. The interval is also needed for the proper working of the energy meter. The duration of measuring pulse does not exceed one millisecond. If we change a number of short counting pulses from 1 to 1000 , we change energy in measuring pulse and in the whole series $10^{3}$ times. Of course, duration of measuring pulse will be changed from 1 microsecond to 1 millisecond with a constant period between pulses. A sensitive pyroelectric energy meter with a time constant of $2-3.10^{-3}$ second measures an average energy from 100 measuring pulses, as it was described in introduction. If we know the number of counting pulses, we can calculate energy of each counting pulse. If the whole series is in interval of $1 \mathrm{sec}$, it can be measured also by standard calorimeter with time constant about 30 sec. A small error of this measurement can be calculated. Overall we can change energy series pulses $10^{5}$ times in this example. Pyroelectric energy meter can be calibrated by standard calorimeter and we have energy-calibrated pulses with low level of energy for other purposes. LED can be used for such operation. The more convenient LED is GaAs unit with wavelength of radiation $910 \mathrm{~nm}$. Of course we can use an averaging energy meter for measuring energy of a single measuring pulse. The number of counting pulses can be increased in this case, but large time constant of measuring device and low repetition rate are needed. It must be a special designed device. But in this case number of counting pulses can be increased to $10^{6}$ and more.

Such method permits to receive any energy levels, corresponding from 1 counting pulse to maximum value. The method permits to check linearity of energy meters and detectors with a time constant longer than the duration of measuring pulse in a wide dynamic range. It is very useful in cases, when we have not calibrated the optical filter, especially in a wide spectral range selective source and energy meter. Sometimes we have not the possibility to measure transmittance and reflectance of optical filters and attenuators in the far infrared region of spectrum, where nonlinear detectors must be used (photo resistor, bolometer and so on.) In this case, linearity of detectors tests from the very beginning and then measurements take place. Counting method is very useful in this case. Infrared semiconductor lasers and LEDs with nanosecond rise time have been recently 
designed. This allows using such source in this region of spectrum as well. We can use pulse-illuminating source for measuring transmittance or reflectance of samples or check detectors and preamplifier linearity.

\section{CALIBRATION BY PHOTODIODE-BASED TRAP DETECTOR}

LED pulse source can be calibrated also by self-calibrating photodiode-based trap detector [1]. Responsivity of trap detector is given in power or radiation flux units. So it is necessary to have some comparison photometer, which can transmit responsivity in power units into responsivity in energy units. A very simple circuit (Fig.1) does this operation. The detector ( $\mathrm{Si}$ photodiode or vacuum diode) is a loaded parallel resistor $R$ and capacitance $C$, which must be precisely measured. Values of $R$ and $C$ are chosen to obtain required time constant $R C$ for precise energy measurements. It must be 3-5 times more than the measurement pulse duration. The voltage on resistor $R$ and capacitance $\mathrm{C}$ is measured by oscilloscope or by peak voltmeter in case of pulse radiation, as well as direct current voltmeter in case of continuous wave (CW) radiation. Responsivity of comparison photometer (Fig.1) in power units is measured in a standard way. Radiation (light) from Lamp, 1, (Fig.2) enters through the entrance slit, 2, of monochromator, 3 , focused by lens, 4. Another lens, 5, behind output slit, 6 , creates image of grating or prism surface, 7 , on the sensitive area of detector, 8 , so as the uniform illumination is ensured over this area. The diaphragm, 9, is mounted both in front of the trap detector and in front of the comparison photometer to be calibrated. The comparison photometer is placed in the same place as the trap detector. The wavelength of monochromator is chosen in the visible range of spectrum (usually $0.5-0.7 \mu \mathrm{m}$ ), where quantum efficiency of the silicon trap detector has the maximum value. The relative responsivity of the comparison photometer detector in spectral range of $0.5-0.95 \mu \mathrm{m}$ must be measured in advance.

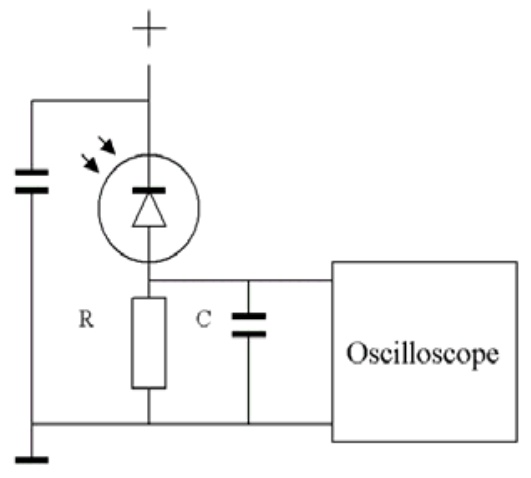

Fig.1 Calibration circuit.

Then, responsivity of comparison photometer detector in power units $S_{p}$ is calculated at $0.91 \mu \mathrm{m}$ wavelength. Responsivity for CW radiation $S_{p}$ is equal to $S_{p}=U_{R} / R \Phi$, where $U_{R}$ is the voltage on resistor $R$ and $\Phi$ is the radiation flux (power) on the input surface of the detector.

In a pulse regime responsivity $S_{q}$ is equal to $S_{q}=C U_{c} / Q$, where $U_{c}$ is the voltage on capacitance $C$ and $Q$ is the energy of measurement pulse of radiation. Relative change of detector responsivity $S_{p}$ and $S_{q}$ shows nonlinearity of light characteristic of the detector. We must have linear regime in all dynamic ranges of measurements. In this case the responsivity $S_{p}$ is equal to the responsivity $S_{q}$ and

$$
\frac{Q}{\Phi}=R C \frac{U_{c}}{U_{R}}
$$

Because all values in (1) except $Q$ are measured, we can calculate energy $Q$. One can also obtain the responsivity $S_{p}$ $(\mathrm{A} / \mathrm{W})=C S_{q}(\mathrm{~V} / \mathrm{J})$ from this equation. The last equation is very useful for energetic calculations.

\section{COUNTING SOURCE APPLICATION}

According to this calibration method the responsivity in term of power is multiplied by time constant $R C$ and as a result one can obtain the responsivity of comparison photometer in terms of energy. The comparison photometer calibrates energy source of radiation with the short pulse duration, which can be considered as $\delta$ - pulse source for this photometer. So we receive small level energy standard for energy meter calibration. Such $\delta$ - pulse source can be the abovementioned source with a single fulfilled pulse. If energy of single $\delta$ - pulse is not enough for some energy meter calibration, the number of fulfilled pulses may be increased, if the time constant of calibrated energy meter is enough. An error of such calibration may be $2-5 \%$ in a wide dynamic range. The most precise error calculation is made on paper [2]. It must be mentioned there are no optical filters or other optical attenuators in use. Therefore there is no problem with a scattering light. In most cases dark conditions are not required for work, because energy meters are not sensitive to CW radiation.

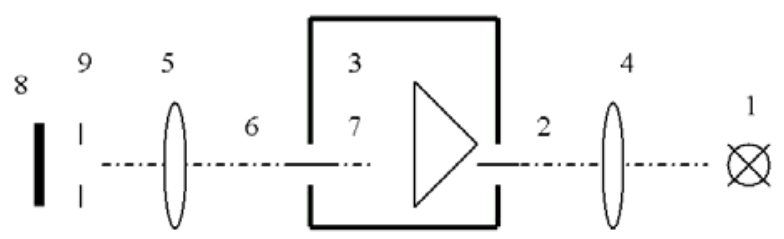

Fig.2 Optical diagram of measuring unit

The described source is acceptable for calibrations in terms of energy, where the form of pulse does not matter. Sometimes rise time of lasers or LEDs are not enough and front of pulse will be delayed. If we use linear parts of dynamic range this circumstance does not matter, so the decay of pulse will be also delayed and common area of pulse will be the same. But if we are not sure we can put another LED with a good rise time in series and compare the ratio of responsivities for both diodes. Current flowing through the diodes will be the same in this case.

If we want to calculate a result of calibration in terms of power, there will be some difficulties. In common case it is necessary to add some coefficient, depending on the form of measuring pulse. There is no problem in case of rectangular pulse. So it is desirable to have illuminating source with rectangular pulse whose duration is a proportional number of fulfilled counting pulses. Then power $\Phi$ can be calculated as $\Phi=Q / t$, where $t$ is rectangular pulse duration. 


\section{EXPERIMENTAL INVESTIGATION AND EQUIPMENT}

We have used GaAs LED in our experimental equipment. A LED was put into pulse generator with constant amplitude of pulse. Radiation of LED was measured by Si photodiode and oscilloscope. The current flowing through the diode can be changed. We used fixed current and then we defined the decreasing top of pulse with increasing of pulse duration. The frequency of pulses was not more then $1 \mathrm{~Hz}$. So we defined an optimal current and duration of pulse. It was current $2 \mathrm{~A}$ in a pulse and pulse duration $1.10^{-2}$ sec for our LED AL107B type. The more powerful diode with rise time of radiation $1.5 .10^{-8}$ sec was chosen. Maximum wavelength of radiation is $910 \mathrm{~nm}$; power in pulse is $60 \mathrm{~mW}$ and maximum energy is $6 \cdot 10^{-4} \mathrm{~J}$. So, low rise time permits escape error under calculation energy of radiation for shortest pulse duration. Power amplifier is used as power supply for this diode, which allows us to receive maximum energy $6.10^{-3} \mathrm{~J}$ for series of pulses and minimum $6.10^{-8} \mathrm{~J}$ for a single pulse, as in the above described example.

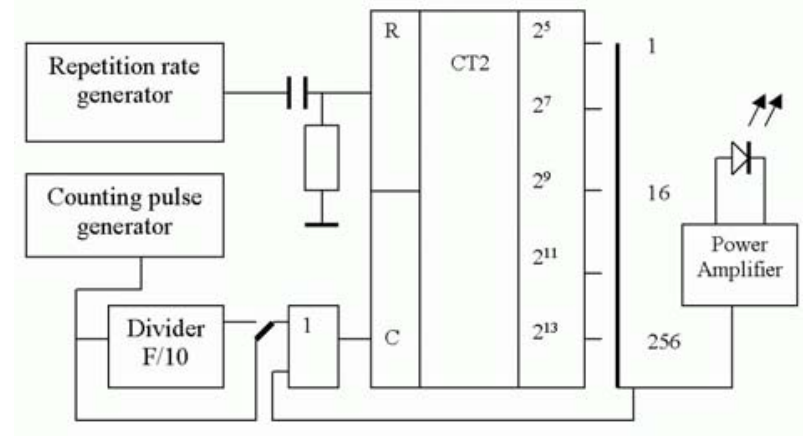

Fig.3 Schematic diagram of experimental unit.

Such LED with corresponding power supply has comparable responsivity with all mentioned energy meters and permits mutual calibration.

One of such possible circuit sources is shown in Fig.3. The monostable multivibrator contains counter divider CT2 with integrated circuit ICs1 (561IE16). The circuit has 2 inputs R and C. Input C is connected to the output "and gate" ICs2 (561LE5). One input of ICs2 is connected to one of the outputs of ICs1. High potential on output ICs1 does not permit the counting pulses from counting pulse generator 1to go through ICs2, until a triggering pulse from repetition rate generator 2 appears. Appearing on one of the outputs, zero potential permits the counter ICs1 to start working. After counting $2^{5}$ counting pulses on the first output ICs1, positive potential stopped counting after corresponding $2^{5}$ pulses. The same operation takes place when input ICs2 is connected to another output ICs2, but output pulse will be 4, 16, 64 and 256 times longer. This pulse goes to power amplifier 3 with LED on its output. Divider 4 decreases frequency of counting pulses and increases duration output measuring pulse. The described monostable multivibrator changes duration of output pulse step by step from 1 to 2560 times. Using divider 4 with more times of dividing, we can increase this number. In experimental circuit, counting pulse generator was stabilized by quartz with frequency $3515 \mathrm{kHz}$. The corresponding output 1 pulse duration is 8.890 microseconds.

Naturally, such unit can be made by using a microprocessor circuit. Usually it is required to have one or few pieces of units. In this case the design of the necessary program is expensive. Microprocessor chip has a lot of pins and it is necessary to use manufacturing technology for PC board and solder it. A common number of soldering points in the described unit is not more than in a microprocessor chip.

\section{CONCLUSIONS}

Possibility of adding part of radiation energy in time allows simplifying the linearity checking of energy meters. Using the described counting source allows us to know precisely parts of energy. It is especially convened in the far infrared region of spectrum where there are no calibrated filters and attenuators of radiation. Sometimes it is difficult to divide calibration and linearity checking, especially in a wide dynamic range, more then $10^{6}$. In this case two standard energy meters are used for linearity checking. The ratio of readouts for standard energy meters is compared with the ratio of readouts for the checking device. Difference between these ratios shows the nonlinearity of the checking device. Simultaneously, calibration takes place in this case automatically.

\section{REFERENCES}

[1] Wolf. W. L (1998) A Publication of SPIE, Bellingham, Washington USA.

[2] Kuvaldin E.V. (2006) Calibration pulse energy and power photometry devices with time conversion. Journal of optical technology volume 73, Number 8, pp. 42-47.

Received December 5, 2009. Accepted April 6, 2010. 